\title{
Myocardial Perfusion Evaluation with T2-Prepared Gradient Echo Blood Oxygen Level Dependent Imaging at 3 Tesla
}

\author{
SM Shea ${ }^{1,2}$, BE Schirf $^{2}$, X Bi ${ }^{1,2}, \mathrm{R}_{\text {Tang }}^{2}$, \\ $\mathrm{B} \mathrm{Lu}^{2}$, RA Omary ${ }^{2}, \mathrm{D} \mathrm{Li}^{1,2}$ \\ ${ }^{1}$ Department of Biomedical Engineering, Northwestern University, Evanston, IL, USA \\ ${ }^{2}$ Department of Radiology, Northwestern University Medical School, Chicago, IL, USA
}

\begin{abstract}
Detecting myocardial perfusion reserve differences using Blood Oxygen Level Dependent (BOLD) MRI may improve at $3 T$ vs 1.5T. Stenosis-model dogs $(n=3)$ were imaged at $3 T$ using a gradient echo sequence (GRE) with T2-preparation for BOLD weighting and without as a control. 3 short-axis slices were acquired at rest and during stenosis and adenosine-induced stress with fluorescent microspheres injected to provide blood flow information. Quantitative measurements showed significant changes for BOLD images (left anterior descending (LAD) or septal vs left circumflex (LCX) regions $=1.20 \pm 0.11 ;$ LAD vs septal regions $=1.03 \pm 0.06$; $p<0.001$ ), but not for control images (LAD or septal vs $L C X$ regions $=1.01 \pm 0.04 ;$ LAD vs septal regions $=$ $0.99 \pm 0.05 ; \quad p=0.09)$. BOLD MR vs microsphere measured flow $(M R=0.053 * S P H E R E+1.03)$ showed good correlation $(R=0.61)$. In conclusion, $3 T$ BOLD imaging was able to identify myocardial perfusion changes.
\end{abstract}

\section{Introduction}

A significant effect of coronary heart disease is reduced myocardial perfusion reserve. Several different imaging modalities are able to assess myocardial perfusion reserve, including nuclear imaging, echocardiography, and Magnetic Resonance Imaging (MRI). However, only MRI has shown the capability to noninvasively acquire other diagnostic information such as ventricular [1] and valve [2] function, tissue viability [3], and coronary morphology [4] without the use of ionizing radiation.

Blood Oxygen Level Dependent (BOLD) MRI is one MR technique that has been used to detect regional changes in myocardial perfusion reserve. The BOLD effect is based on the concentration of the paramagnetic molecule, deoxyhemoglobin, in the blood. An increase in blood flow in the myocardium without an increase oxygen consumption, as is seen after adenosine or dipyridamole infusion, will result in an increase in myocardial $\mathrm{T} 2 *$ and $\mathrm{T} 2$ relaxation times due to a decrease in deoxyhemoglobin. Previous studies at $1.5 \mathrm{~T}$, the most common magnet strength for clinical scanners, have shown that changes in blood flow in myocardium can be detected using the BOLD effect [5-7]. However, signalto-noise ratios (SNR) have been low, and T2 and T2* signal changes have been small, thereby limiting the effectiveness of BOLD techniques to detect small flow differences. A possible solution is imaging at 3T. SNR and the susceptibility of deoxyhemoglobin have been shown to increase with field strength [8-10], which should amplify BOLD signal changes in the heart compared to $1.5 \mathrm{~T}$. This should improve the detection of regional flow differences in the myocardium using BOLD sequences.

The purpose of this study was to examine the feasibility of BOLD myocardial imaging in a commercial 3T whole body scanner using a dog stenosis model. A T2prepared gradient echo (GRE) sequence was chosen for BOLD imaging at $3 \mathrm{~T}$ for several reasons. While signal and the BOLD effect both increase at higher field strengths, magnetic inhomogeneities increase as well. $\mathrm{T} 2 *$-weighted sequences used for cardiac BOLD imaging have exhibited significant artifacts in the posterior-septal and posterior-lateral regions of the heart, even at 1.5T, due to local field inhomogeneities. T2-weighted sequences are less affected by field inhomogeneity. The short-TR GRE sequence structure is also optimal for cardiac imaging because it is more resistant to motion and flow artifacts than typical $\mathrm{T} 2 *$-weighted sequences or a T2-weighted turbo spin-echo sequence.

\section{Methods}

Three mongrel dogs were operated on and studied with approval from our university's Animal Care and Use Committee. A thoracotomy was performed in order to place two devices on the left circumflex artery (LCX): an external hydraulic occluder capable of inducing a reversible stenosis of variable size; and a Doppler flow probe to measure relative flow in the LCX downstream from the occluder. Several catheters were also placed in 
the aorta and the left and right atria for the delivery of saline, adenosine, and fluorescent microspheres. The chest was closed and the dogs were allowed to recover for 7 days before imaging.

A segmented, ECG-triggered, 2D GRE sequence with T2-preparation was used for BOLD imaging. The T2preparation was modified slightly from the original design scheme presented by Brittain et al. [11]. Four, single $180^{\circ}$ yF pulses replaced the four, $180^{\circ}$ composite pulse structures $\left(90^{\circ}{ }_{\mathrm{x}}: 180^{\circ} \mathrm{y}\right.$ : $\left.-90^{\circ}\right)$ and a single $-90^{\circ}{ }_{\mathrm{x}}$ pulse was used for magnetization flip-back instead of the $270^{\circ}{ }_{x}:-360^{\circ}{ }_{x}$ composite pulse in order to meet specific absorption rate (SAR) limits for 3T imaging. The parameters for each T2-prepared GRE sequence were as follows: TR/TE $=4.0 / 1.6 \mathrm{~ms}$, flip angle $=15^{\circ}$, readout bandwidth $=340 \mathrm{~Hz} /$ pixel, T2-preparation time $=44 \mathrm{~ms}$, FOV $=150 \times 300 \mathrm{~mm}^{2}$, matrix $=99 \times 256$; lines per heartbeat $=9$, slice thickness $=5 \mathrm{~mm}$, number of averages $=3$, trigger delay $=350-450 \mathrm{~ms}$ (adjusted to acquire image data during mid-diastole), breath-hold duration = $15-20$ s. Asymmetric sampling was employed to reduce TR with the echo center occurring at the $64^{\text {th }}$ point in a 256-point readout period. Phase-encoding lines were acquired centrically. GRE images without T2-preparation were collected using the same parameters to serve as a control for the T2-prepared BOLD images. An ECGtriggered, 2D saturation recovery (SR) gradient echo sequence was used for first-pass imaging. Imaging parameters were as follows: TR/TE $=2.8 / 1.4 \mathrm{~ms}$, flip angle $=20^{\circ}$, readout bandwidth $=490 \mathrm{~Hz} /$ pixel, FOV $=$

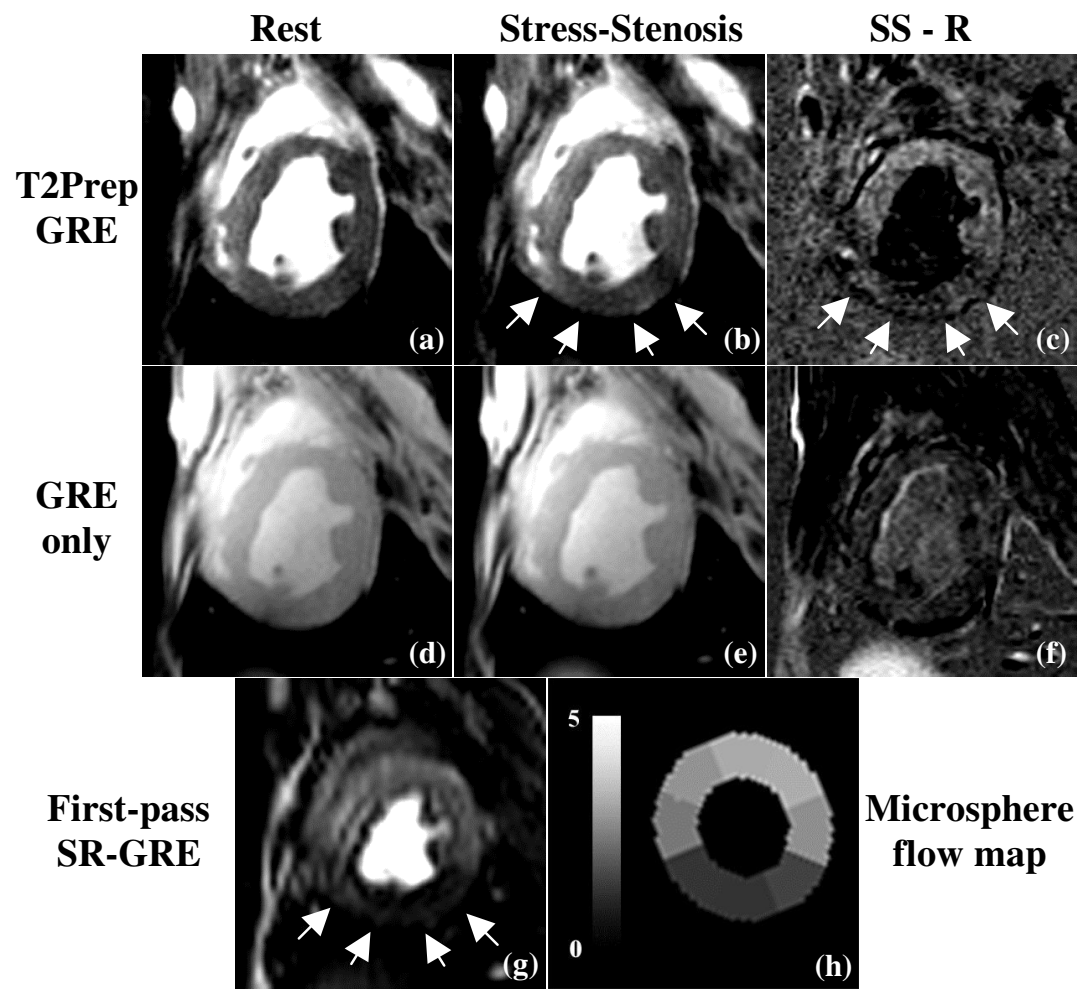

$150 \times 300 \mathrm{~mm}^{2}$, matrix $=64 \times 128 ;$ slice thickness $=5 \mathrm{~mm}$, slices per heartbeat $=3$, breath-hold duration $=20-30 \mathrm{~s}$.

Imaging was carried out on a 3T Siemens Trio scanner (Siemens Medical Solutions, Erlangen, Germany). First, 3 short-axis slices were acquired during rest conditions using the GRE sequence with and without T2preparation. Fluorescent microspheres were also injected into the left atrium in order to provide regional myocardial perfusion information. Next, adenosine was administered intravenously at $0.14 \mathrm{mg} / \mathrm{kg} / \mathrm{min}$ to produce an increase in coronary blood flow from vasodilation. This was confirmed using the Doppler probe. Then, the LCX was stenosed using the occluder and images were reacquired. Microspheres were injected again to determine perfusion. Afterwards, the pressure was released in the occluder and flow was allowed to return to baseline conditions. The same imaging and microsphere protocols were then repeated using different stenosis sizes by varying the inflation of the occluder. During the last stress-stenosis condition of each study, first-pass SR gradient echo images were collected for visual comparison with the GRE images with and without T2preparation. $\quad 0.075 \mathrm{mmol} / \mathrm{kg}$ of gadopentetate dimeglumine (Magnevist ${ }^{\circledR}$, Berlex Laboratories, Inc., Montville, NJ) was injected via IV followed by a $10 \mathrm{cc}$ saline flush during data collection. At the end of experiments, animals were euthanized and their hearts were excised and sectioned for microsphere flow analysis. This procedure and the subsequent analysis were done in a similar manner as was presented in Fieno et al. [12].
Figure 1: Comparison of T2-prepared GRE, GRE-only, first-pass SR-GRE, and microsphere flow map images. The first row contains T2-prepared GRE BOLD images acquired at rest (a) and during stress-stenosis (b) and a subtraction image of those images at stress-stenosis and rest (c). The stressstenosis T2-prepared GRE image (b) shows a reduced region of perfusion (arrows) in the myocardium fed by the stenosed LCX that is not seen in the rest image (a). This can be seen even more clearly in the subtraction image (c). The second rows shows GRE-only images acquired at rest (d) and during stressstenosis (e) and a subtraction image (f) of the first two. The stress-stenosis (e) and subtraction (f) GRE-only images do not show any signal changes in the LCX-fed myocardium. The first-pass SR-GRE image (g) and microsphere flow map (h) show similar reduced regions of perfusion to the T2-prepared GRE stress-stenosis (b) and subtraction (c) images. (The microsphere flow map was generated by calculating flow ratios in each segment in relation to one reference segment. Scale indicates black for 0 flow and white for a 5-fold flow ratio.) 
For GRE images with and without T2-preparation signal intensity was measured in 3 different myocardial territories fed by the left anterior descending (LAD), the septal (SEPT), and the LCX arteries, respectively. Stressstenosis measurements were normalized to rest measurements to account for coil differences. Three regional comparisons were then made by dividing the normalized signal intensities as follows: LAD/LCX, SEPT/LCX, and LAD/SEPT. Results were then matched to microsphere results based on anatomical landmarks. Mean and standard deviations were calculated for these comparisons (with the LAD/LCX and SEPT/LCX lumped into one group) and analyzed for significant differences using a two-tailed student's t-test assuming unequal variances. MR signal changes and microsphere flow changes were also analyzed using a linear mixed effect regression model.

\section{Results}

9 different stress-stenosis studies with microspheres were performed on the three dogs. For all studies, 3 shortaxis slices were acquired with T2-prepared GRE. In 2 studies, only 1 short-axis slice was acquired with GRE; all other studies had 3 slices collected with GRE.

During stress-stenosis, T2-prepared GRE BOLD images showed substantial signal changes between the LCX-fed myocardial territory and the territory fed by the LAD and septal arteries (Fig. 1b and 1c, Fig. 2a-2c). GRE images without T2-preparation did not show similar signal changes (Fig. 1d-1f), indicating that BOLDinduced $\mathrm{T} 2$ changes were responsible for signal differences. Comparison with the first-pass images (Fig. 1g, Fig. 2d-2f) and microspheres (Fig. 1h) showed similar regions of reduced perfusion.

Results of the signal comparisons are listed in Table 1. Microsphere flow measurements indicated that significant perfusion changes occurred in the myocardium fed by the stenosed LCX artery, while the LAD and SEPT myocardial areas were not adversely affected. T2prepared GRE measurements mirrored the microsphere results, with the comparisons to the LCX region showing significant perfusion changes. Control GRE images did not show any significant signal changes between the LAD, SEPT, and LCX myocardial regions. Normalized MR signal change versus normalized microsphere flow change was plotted in Figure 3 for GRE data with and without T2-preparation. The linear mixed effect regression model $(\mathrm{MR}=0.053 * \mathrm{SPHERE}+1.03)$ for $\mathrm{T} 2$ prepared GRE BOLD data showed good correlation between MR signal changes and microsphere measured blood flow changes $(\mathrm{R}=0.61)$. As expected, GRE data $(\mathrm{MR}=0.0057 *$ SPHERE +1.00$)$ showed poor correlation $(\mathrm{R}=0.18)$, indicating no relationship between MR signal and flow changes.
Table 1

\begin{tabular}{lcccc}
\hline & LAD or SEPT & & $\underline{\text { LAD }}$ & $\mathrm{p}$ \\
& LCX & & SEPT & TEn \\
T2prep sphere & $2.55 \pm 1.08$ & & $1.23 \pm 0.34$ & $<<0.01$ \\
GRE sphere & $2.45 \pm 1.13$ & & $1.24 \pm 0.33$ & $<<0.01$ \\
T2prep MR & $1.20 \pm 0.11$ & $1.03 \pm 0.06$ & $<<0.01$ \\
GRE MR & $1.01 \pm 0.04$ & $0.99 \pm 0.05$ & 0.09 \\
\hline
\end{tabular}

\section{Discussion and conclusions}

Regions of reduced myocardial perfusion could be identified in T2-prepared GRE BOLD images during stress-stenosis. Regional signal differences were even more clearly delineated in difference images (stressstenosis - rest). Quantitative results also showed significant signal changes in the myocardium fed by the LCX when compared to other regions of the heart. In comparison, reduced perfusion regions were not identifiable in GRE images without T2-preparation and measurements of signal changes in those images did not show significant differences between myocardial regions

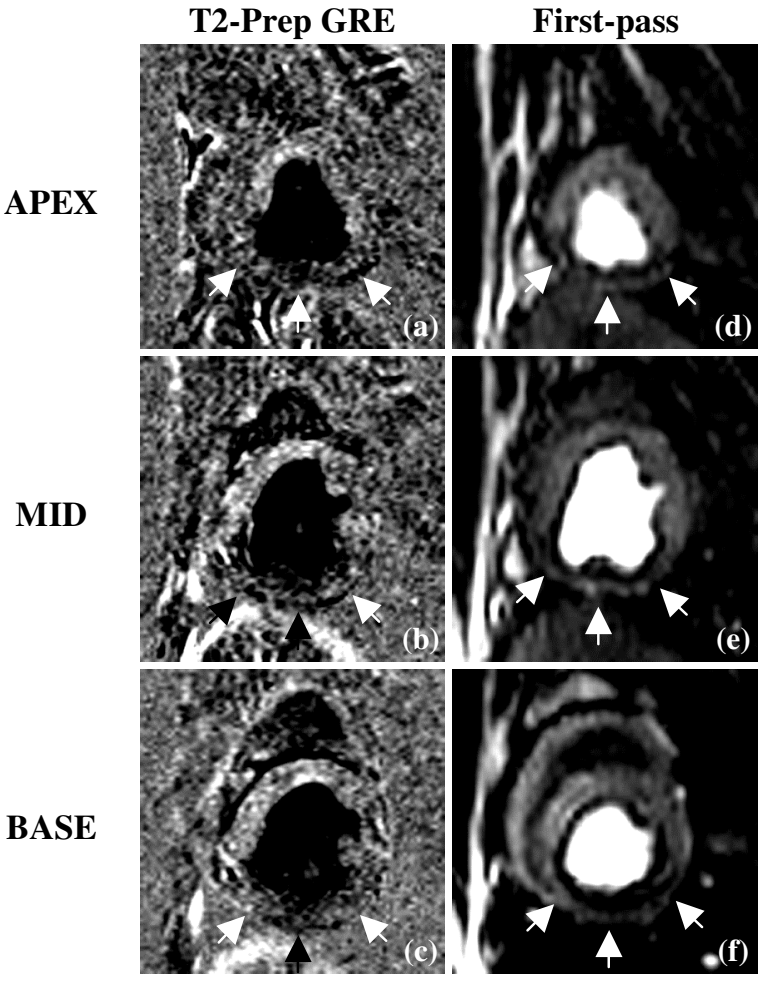

Figure 2: Three T2-prepared GRE difference images (a-c) showing signal changes in the LCX territory (arrows) due to reduced perfusion from stenosis. First-pass images (d-f) showed similar reduced perfusion regions (arrows), although with lower resolution. 


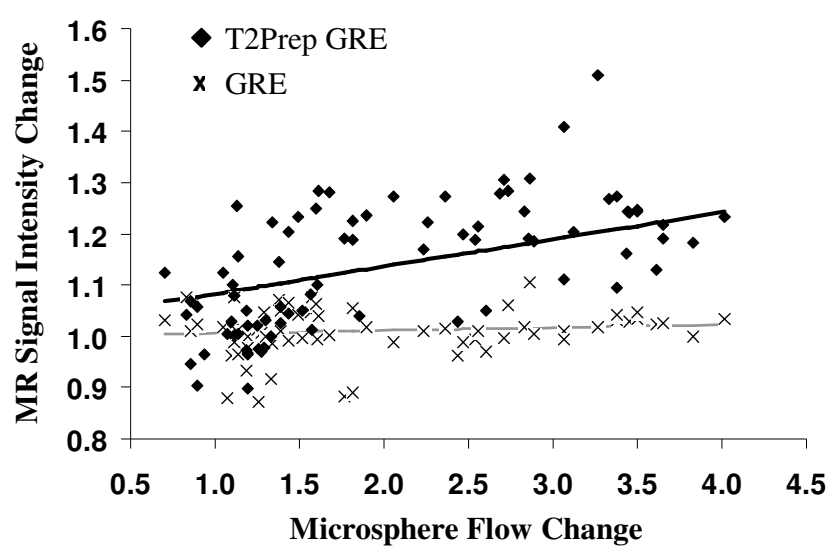

Figure 3: Plot of normalized microsphere flow change versus normalized MR signal intensity change. T2-prepared GRE data is indicated by solid diamonds, while GRE-only data is indicated by x's. The solid black line represents the fitted model for T2-prepared GRE, while the gray line represents the fitted model for GRE.

fed by the LAD, LCX, and SEPT arteries. From this, we can conclude that signal changes were the result of T2 changes within the myocardium, rather than T1-related, through-plane flow effects. The T2 changes most likely resulted from the BOLD effect, whereby the level of deoxyhemoglobin decreased in LAD and SEPT-fed myocardial regions due to adenosine-induced increased perfusion while the level of deoxyhemoglobin remained constant or increased in stenosed LCX-fed myocardium.

Problems did occur with BOLD imaging at 3T. Signal variation across some images was significant possibly due to coil deficiencies. Off-resonance artifacts were also present in some cases, even with the use of T2-weighted sequences. This may explain the large variation around the regression line. Future studies may benefit from better coil design and enhanced shimming techniques to reduce artifacts.

Overall, MR signal changes correlated well with microsphere measured flow changes. MR signal changed by roughly $5 \%$ for a $2: 1$ perfusion change. A previous study by Wright et al. [7] using a similar T2-prepared GRE sequence in a LCX catheter dog model at $1.5 \mathrm{~T}$ showed an average signal change of $17 \%$ for an average perfusion increase of $320 \%$ (a 4.2:1 flow change). For our data at 3T, a $320 \%$ increase in perfusion would result in a $25 \%$ MR signal increase (based on the linear mixed effect model). Based on this, we can conclude that imaging at 3T showed a larger sensitivity to the BOLD effect, which may result in the ability to identify smaller myocardial perfusion changes.

\section{References}

[1] Lorenz CH, Walker ES, Morgan VL, Klein SS, Graham TP, Jr. Normal human right and left ventricular mass, systolic function, and gender differences by cine magnetic resonance imaging. J Cardiovasc Magn Reson 1999;1(1):721.

[2] Higgins CB, Wagner S, Kondo C, Suzuki J, Caputo GR. Evaluation of valvular heart disease with cine gradient echo magnetic resonance imaging. Circulation 1991;84(3 Suppl):I198-207.

[3] Kim RJ, Wu E, Rafael A, Chen EL, Parker MA, Simonetti $\mathrm{O}$, et al. The use of contrast-enhanced magnetic resonance imaging to identify reversible myocardial dysfunction. $\mathrm{N}$ Engl J Med 2000;343(20):1445-1453.

[4] Kim WY, Danias PG, Stuber M, Flamm SD, Plein S, Nagel $\mathrm{E}$, et al. Coronary magnetic resonance angiography for the detection of coronary stenoses. $\mathrm{N}$ Engl $\mathrm{J}$ Med 2001;345(26):1863-1869.

[5] Niemi P, Poncelet BP, Kwong KK, Weisskoff RM, Rosen BR, Brady TJ, et al. Myocardial intensity changes associated with flow stimulation in blood oxygenation sensitive magnetic resonance imaging. Magn Reson Med 1996;36(1):78-82.

[6] Li D, Dhawale P, Rubin PJ, Haacke EM, Gropler RJ. Myocardial signal response to dipyridamole and dobutamine: demonstration of the BOLD effect using a double-echo gradient-echo sequence. Magn Reson Med 1996;36(1):16-20.

[7] Wright KB, Klocke FJ, Deshpande VS, Zheng J, Harris $\mathrm{KR}$, Tang R, et al. Assessment of regional differences in myocardial blood flow using T2-weighted 3D BOLD imaging. Magn Reson Med 2001;46(3):573-588.

[8] Edelstein WA, Glover GH, Hardy CJ, Redington RW. The intrinsic signal-to-noise ratio in NMR imaging. Magn Reson Med 1986;3(4):604-618.

[9] Turner R, Jezzard P, Wen H, Kwong KK, Le Bihan D, Zeffiro $\mathrm{T}$, et al. Functional mapping of the human visual cortex at 4 and 1.5 tesla using deoxygenation contrast EPI. Magn Reson Med 1993;29(2):277-279.

[10] Gati JS, Menon RS, Ugurbil K, Rutt BK. Experimental determination of the BOLD field strength dependence in vessels and tissue. Magn Reson Med 1997;38(2):296-302.

[11] Brittain JH, Hu BS, Wright GA, Meyer CH, Macovski A, Nishimura DG. Coronary angiography with magnetizationprepared T2 contrast. Magn Reson Med 1995;33(5):689696.

[12] Fieno DS, Shea SM, Li Y, Harris KR, Finn JP, Li D. Myocardial perfusion imaging based on the blood oxygen level-dependent effect using T2-prepared steady-state freeprecession magnetic resonance imaging. Circulation 2004;110(10):1284-1290.

Address for correspondence

Debiao Li, Ph.D.

448 East Ontario Street, Suite 700

Chicago, IL 60611

E-mail: $\underline{\text { d-li2@ @orthwestern.edu }}$ 